\title{
Massive binaries as progenitors for stellar explosions
}

\author{
Selma de Mink \\ Anton Pannenkoek Astronomical Institute, University of Amsterdam, 1090 GE Amsterdam, \\ The Netherlands \\ email: S.E.deMink@uva.nl
}

\begin{abstract}
The majority of young massive stars are found in close binary systems. Recently, dedicated observingcampaigns have provided strong constraints on the binary fraction as well as the distribution of the parameters thatcharacterize the binary systems: the masses of both components, the orbital period and eccentricities. Most strikinglythese findings imply that the majority of massive stars experience strong interaction (roche lobe overflow, a commonenvelope phase and or a merger) with a binary companion before their final explosion. I will discuss recent resultsfrom detailed binary star models and population synthesis models.
\end{abstract}

